\title{
Low FAT4 expression is associated with a poor prognosis in gastric cancer patients
}

\author{
Xiaoting Jiang ${ }^{1}$, Zhengchuang Liư ${ }^{1}$, Yingjie Xia ${ }^{1}$, Jungang Luo ${ }^{1}$, Ji Xu ${ }^{2}$, Xujun $\mathrm{He}^{1}$ \\ and Houquan Tao ${ }^{1,2}$ \\ ${ }^{1}$ Key Laboratory of Gastroenterology of Zhejiang Province, Zhejiang Provincial People's Hospital, People's Hospital of \\ Hangzhou Medical College, Hangzhou 310014, Zhejiang, China \\ ${ }^{2}$ Department of Surgery, Zhejiang Provincial People's Hospital, People's Hospital of Hangzhou Medical College, Hangzhou \\ 310014, Zhejiang, China \\ Correspondence to: Houquan Tao, email: taohouquan2008@aliyun.com \\ Xujun He, email: hxj0105099@163.com
}

Keywords: FAT4; gastric cancer; prognosis; methylation; distant metastasis

Received: April 15, $2017 \quad$ Accepted: December 11, $2017 \quad$ Published: December 26, 2017

Copyright: Jiang et al. This is an open-access article distributed under the terms of the Creative Commons Attribution License 3.0 (CC BY 3.0), which permits unrestricted use, distribution, and reproduction in any medium, provided the original author and source are credited.

\section{ABSTRACT}

In this study, we investigated the role of Fat atypical cadherin 4 (FAT4) in gastric cancer (GC) progression. Immunohistochemical analysis showed lower FAT4 expression in tumor tissues from GC patients than in normal gastric epithelium. Lower FAT4 expression was associated with poor prognosis, tumor size and invasion, and lymph node and distant metastases. Multivariate analysis showed that TNM stage, lymph node and distant metastases, Lauren classification, and FAT4 expression were independent prognostic factors in GC. Methylation-specific PCR analysis showed increased FAT4 promoter methylation in GC tumor tissues and cell lines. Higher FAT4 promoter methylation was associated with low FAT4 expression and a poor prognosis. BGC-823 cells showed increased FAT4 expression upon treatment with 5-azacytidine, demethylating agent. FAT4 knockdown in BGC-823 cells led to increased cell proliferation, migration and invasiveness. Moreover, xenografts of BGC823 cells with FAT4 knockdown showed enhanced tumor growth and metastasis in nude mice. These findings demonstrate that low FAT4 expression is associated with a poor prognosis in GC patients.

\section{INTRODUCTION}

Despite recent developments in the surgical techniques and improved efficacy of anticancer drugs, mortality rates of gastric cancer (GC) patients remains very high. The overall 5-year survival rate of GC is about $40 \%$ because of late diagnosis (stage III and IV), which is associated with lymph node and distant metastasis [1]. Therefore, new prognostic and therapeutic biomarkers are necessary to improve the survival of GC patients.

The Fat gene family was originally identified in Drosophila as a member of the cadherin super-family with tumor suppressor functions [2,3]. It regulates cell proliferation and planar cell polarity during Drosophila development by the Hippo signaling pathway $[4,5]$. They encode a type 1 trans-membrane protein with 34 cadherin repeats, 4 epidermal growth factor (EGF)-like repeats, a transmembrane domain and a cytoplasmic domain that is distinct from the classical cadherin proteins $[6,7]$. In humans, four members of the Fat family have been identified, namely, FAT1, FAT2, FAT3 and FAT4, which are structurally similar to the Drosophila Fat protein [3]. In mammals, FAT4 is the true structural ortholog of the Drosophila FAT, whereas FAT1, FAT2 and FAT3 are closely related to the Drosophila Fat-like 8 (Fatl-8). FAT4 mediates key developmental functions such as planar cell polarity and regulates the Hippo signaling pathway, which controls the size of the organs $[3,4]$. 
Whole-exome sequencing revealed that TP53, PIK3CA and ARIDIA were critical cancer driving genes in $\mathrm{GC}$ and cell adhesion genes were frequently mutated [8]. Genomic mutations (5\%) and deletions (4\%) in FAT4 were observed in GC, whereas FAT4 silencing decreased cellular adhesion and increased migration and invasion of GC cells [8]. FAT4 suppresses growth and invasion in breast [9] and GC cancers [10-12] by activating the Yes-associated protein (YAP) and/or WNT signaling and silencing the epigenetic modifier proteins. However, the definitive role of FAT4 in GC distant metastases remains unknown. Therefore, in this study, we investigated the role of FAT4 in GC progression and prognosis.

\section{RESULTS}

\section{FAT4 expression is associated with poor prognosis in GC patients}

Table 1 shows the association between FAT4 expression and clinicopathological features based on IHC analysis of 449 clinical GC tissue microarray (TMA) samples. FAT4 staining was exclusively cytoplasmic in normal gastric epithelial cells and few cases of tumor cells (Figure 1). Low FAT4 expression was observed in GC tissues than in adjacent noncancerous tissues (Figure 1A1F). FAT4 expression was detected in 263 (58.6\%) out of $449 \mathrm{GC}$ tumor samples analyzed. FAT4 expression reduced from $75.9 \%$ to $32.4 \%$ in the patients with lymphatic metastasis with increasing $\mathrm{N}$ grades (N0 to N4 grades). FAT4 expression decreased from $89.3 \%$ to $20.3 \%$ with increasing TNM grades (TNM Grade I-IV, $P<0.05$ ). FAT4 expression was lower in GC patients with lymph node metastasis $(48.4 \%$, or $137 / 283)$ than patients without lymph node metastasis $(75.9 \%$, or $126 / 166, P<0.05)$. FAT4 expression was lower in patients with distant metastasis $(20.3 \%$, or $13 / 64)$ than patients without distant metastasis $(64.9 \%$, or $250 / 385 ; P<0.05)$. Lower FAT4 expression correlated with tumor size, tumor invasion depth (T Grade) and vascular invasion $(P<0.05$, Table 1$)$. FAT4 expression was not associated with age, sex, location, differentiation, Lauren classification and histological type of GC $(P>0.05$, Table 1).

\section{FAT4 expression is associated with GC prognosis}

We analyzed if FAT4 expression was associated with GC prognosis. The median survival time was lower for FAT4 negative patients than in FAT4 positive patients (34. $00 \pm 1.84$ months vs. $49.00 \pm 1.96$ months, $\mathrm{P}<0.01$ ). The 5-year survival rate of the FAT4 negative patients was lower than that of FAT4 positive patients $(9.1 \%$ vs. $33.5 \%$, $P<0.05$, Figure $1 \mathrm{~K}$ ). Cox multivariate analysis showed that TNM stages, distant and lymph node metastasis, Lauren classification and FAT4 expression were independent prognostic factors in GC (Table 2).

\section{FAT4 mRNA levels are reduced in GC patients with distant metastasis}

Next, we analyzed FAT4 mRNA expression in 173 paired GC tissue samples. FAT4 was down-regulated in the tumors than adjacent noncancerous tissues (5.97E-04 $\pm 1.02 \mathrm{E}-03$ vs $9.29 \mathrm{E}-04 \pm 1.21 \mathrm{E}-03, P<0.05$, Figure 1L). We classified the patient samples $(\mathrm{n}=179)$ based on lymph node $(\mathrm{N})$ and distant metastases $(\mathrm{M})$ status into three groups namely, group $1(\mathrm{~N} 0, \mathrm{M} 0, \mathrm{n}=50)$, group $2(\mathrm{~N}>1, \mathrm{M} 0, \mathrm{n}=64)$ and group $3(\mathrm{~N}>1, \mathrm{M} 1, \mathrm{n}=59)$. FAT4 expression was higher in group 1 than in group $3(1.17 \mathrm{E}-$ $03 \pm 0.0013$ vs. $2.01 \mathrm{E}-04 \pm 0.0013$; Figure $1 \mathrm{~L}, P<0.05$ ). FAT4 expression was similar in cancer tissue and adjacent noncancerous tissues in groups 1 (1.17E- $03 \pm 0.0013 v s$. $1.44 \mathrm{E}-03 \pm 0.0016$, Figure $1 \mathrm{~L}, P=0.185)$ and $2(5.12 \mathrm{E}-$ $04 \pm 0.00097$ vs. $7.53 \mathrm{E}-04 \pm 0.00093$, Figure $1 \mathrm{~L}, P=0.09$ ). FAT4 expression was lower in cancer tissues from group 2 patients than group 1 patients $(5.12 \mathrm{E}-04 \pm 0.00097 v s$. $1.17 \mathrm{E}-03 \pm 0.0013$, Figure $1 \mathrm{~L}, P<0.05)$. Moreover, FAT4 expression was lower in cancer tissues than adjacent noncancerous tissues in group 3 patients $(2.01 \mathrm{E}-$ $04 \pm 0.00024$ vs. $6.85 \mathrm{E}-04 \pm 0.0008$, Figure $1 \mathrm{~L}, P<0.05$ ).

The IHC and qRT-PCR data showed that FAT4 expression was higher at the early stages of the GC progression ( $\mathrm{N} 0$ or $\mathrm{T} 1+\mathrm{T} 2$ grade), but gradually decreased during lymph node and distant metastatic events (from group1 to group3, Figure 1L). Low FAT4 expression during cancer metastasis was associated with decreased survival (Figure 1K).

\section{FAT4 promoter methylation is associated with poor prognosis in GC}

Next, we analyzed FAT4 methylation status in 173 paired GC tissue samples to determine if methylation was associated with low FAT4 expression and GC progression. We identified a $\mathrm{CpG}$ island region of approximately 4.5 $\mathrm{kb}$ in the human FAT4 gene promoter region (Figure 2A). We analyzed the methylation status of the FAT4 promoter region in paired GC and adjacent noncancerous tissues by methylation specific PCR (MSP) analysis. We found that $31.21 \%$ (54/173) of GC tissues were methylated (Table 3 and Figure 2B). FAT4 was methylated in AGS, MKN-28 and $\mathrm{MKN}-45$ GC cancer cell lines and unmethylated in the normal GES-1 gastric epithelial cells (Figure 2C). We performed pyrosequencing on $36 \mathrm{GC}$ tissue samples and GC cell lines to verify the results from the MSP method. After re-extraction and quantification, we obtained $32 \mathrm{GC}$ tissues and 6 cell lines for analysis. Four cases (No.7, 23, 29 and 36) had low quality DNA and hence could not be analyzed. We sequenced $6 \mathrm{CpG}$ sites and determined the mean methylation percentage of each site relative to the corresponding matched noncancerous tissue. We classified the specimens based on mean methylation percentages into low $(0-5 \%)$ and high $(>5 \%)$ methylated groups 
Table 1: Association between FAT4 expression and clinicopathological factors

\begin{tabular}{|c|c|c|c|c|}
\hline \multirow{2}{*}{ Clinical parameters } & \multicolumn{3}{|c|}{ FAT4 } & \multirow{2}{*}{$P$} \\
\hline & Positive & Negative & $t / \chi^{2}$ & \\
\hline Age(yrs) & $58.35 \pm 12.11$ & $60.72 \pm 12.70$ & 1.995 & 0.872 \\
\hline Gender & & & 0.000 & 0.996 \\
\hline Male & $188(71.5 \%)$ & $133(28.5 \%)$ & & \\
\hline Female & $75(71.5 \%)$ & $53(28.5 \%)$ & & \\
\hline Location & & & 5.227 & 0.073 \\
\hline Proximal & $26(44.8 \%)$ & $32(55.2 \%)$ & & \\
\hline Middle & $104(61.2 \%)$ & $66(38.8 \%)$ & & \\
\hline Distal & $133(60.2 \%)$ & $88(39.8 \%)$ & & \\
\hline Size & & & 15483 & 0.000 \\
\hline$\geq 5 \mathrm{~cm}$ & $91(47.9 \%)$ & $99(52.1 \%)$ & & \\
\hline$<5 \mathrm{~cm}$ & $172(66.4 \%)$ & $87(33.6 \%)$ & & \\
\hline Histology type & & & 1.448 & 0.694 \\
\hline Papillary adenocarcinoma & $9(56.3 \%)$ & $7(43.7 \%)$ & & \\
\hline Tubular adenocarcinoma & $195(57.9 \%)$ & $142(42.1 \%)$ & & \\
\hline Mucinous adenocarcinoma & $15(53.6 \%)$ & $13(46.4 \%)$ & & \\
\hline Signet-ring cell carcinoma & $44(64.7 \%)$ & $24(35.3 \%)$ & & \\
\hline Lauren classification & & & 0.616 & 0.432 \\
\hline Diffuse type & $161(60.1 \%)$ & $107(39.9 \%)$ & & \\
\hline Intestinal type & $102(56.4 \%)$ & $79(43.6 \%)$ & & \\
\hline Differentiation & & & 2.075 & 0.557 \\
\hline Well & $9(69.2 \%)$ & $4(30.8 \%)$ & & \\
\hline Moderately & $77(58.3 \%)$ & $55(41.7 \%)$ & & \\
\hline Poorly & $175(57.9 \%)$ & $127(42.1 \%)$ & & \\
\hline Undifferentiation & $2(100 \%)$ & $0(0.0 \%)$ & & \\
\hline Invasion Depth (T Grade) & & & 52.860 & 0.000 \\
\hline $\mathrm{T} 1$ & $52(89.7 \%)$ & $6(10.3 \%)$ & & \\
\hline $\mathrm{T} 2$ & $84(71.8 \%)$ & $33(28.2 \%)$ & & \\
\hline $\mathrm{T} 3$ & $120(48.4 \%)$ & $128(51.6 \%)$ & & \\
\hline $\mathrm{T} 4$ & $7(36.8 \%)$ & $19(63.2 \%)$ & & \\
\hline Lymphatic Metastasis (N Grade) & & & 53.868 & 0.000 \\
\hline N0 & $126(75.9 \%)$ & $40(24.1 \%)$ & & \\
\hline N1 & $46(66.7 \%)$ & $23(33.3 \%)$ & & \\
\hline N2 & $57(52.3 \%)$ & $52(47.7 \%)$ & & \\
\hline N3 & $34(32.4 \%)$ & $71(67.6 \%)$ & & \\
\hline
\end{tabular}

(Continued) 


\begin{tabular}{|c|c|c|c|c|}
\hline \multirow{2}{*}{ Clinical parameters } & \multicolumn{3}{|c|}{ FAT4 } & \multirow{2}{*}{$\boldsymbol{P}$} \\
\hline & Positive & Negative & $t / \chi^{2}$ & \\
\hline Distant metastasis (M Grade) & & & 45.033 & 0.000 \\
\hline M0 & $250(64.9 \%)$ & $135(35.1 \%)$ & & \\
\hline M1 & $13(19.7 \%)$ & $51(80.7 \%)$ & & \\
\hline TNM Stages & & & 97.426 & 0.000 \\
\hline I & $92(89.3 \%)$ & $11(10.7 \%)$ & & \\
\hline II & $107(67.7 \%)$ & $53(32.3 \%)$ & & \\
\hline III & $51(41.8 \%)$ & $71(58.2 \%)$ & & \\
\hline IV & $13(20.3 \%)$ & $51(79.7 \%)$ & & \\
\hline Lymphatic metastasis & & & 32.594 & 0.000 \\
\hline Yes & $137(48.4 \%)$ & $146(51.6 \%)$ & & \\
\hline No & $126(75.9 \%)$ & $40(24.1 \%)$ & & \\
\hline Vascular invasion & & & 29.396 & 0.000 \\
\hline No & $135(73.7 \%)$ & $48(26.3 \%)$ & & \\
\hline Yes & $128(48.1 \%)$ & $138(51.9 \%)$ & & \\
\hline
\end{tabular}

All cases were classified according to the World Health Organization's (2010) pathological classification of gastric cancer. Invasion Depth (T Grade) grade T1 includes T1a and T1b, T4 includes T4a and T4b. Lymphatic Metastasis (N Grade) grade N3 includes N3a and N3b. TNM grade I includes Ia and Ib, TNM grade II includes IIa and IIb, TNM grade III includes IIIa, IIIb and IIIc.

(Supplementary Figure 1). As shown in Supplementary Figure 2B, the pyrosequencing data confirmed the MSP results in high methylation samples (Nos. 2, 4, 11-18; Supplementary Figures 1 and 2) as well as low methylation samples (Nos. 6, 9, 10 and 32; Supplementary Figures 1 and 2). FAT4 promoter showed higher methylation percentage in AGS, MKN-28 and MKN-45 GC cancer cell lines (between 10\% and 20\%) than 7901, BGC-823, HGC27 and the normal GES-1 gastric epithelial cells, which showed no methylation (Supplementary Figure 3A). These data demonstrated that FAT4 promoter was methylated in advanced GC cases.

\section{Association between $F A T 4$ promoter methylation status and GC clinicopathological factors}

We treated GC tumor cell lines AGS, MKN-28 and MKN-45 with $1 \mu \mathrm{M}$ of 5-aza-2'-deoxycytidine (5-Aza-dC) for $48 \mathrm{~h}$ and demonstrated increased expression of FAT4 mRNA (Supplementary Figure 4). This indicated that methylation of the FAT4 promoter silenced the expression of FAT4.

Nearly $31.2 \%(54 / 173)$ of the GC patients exhibited FAT4 methylation. Next, we analyzed the association between FAT4 methylation and expression with the clinicopathological parameters and prognosis of GC. We observed that FAT4 methylation status was associated with
Lauren classification, invasion depth (T Grade), lymphatic metastasis ( $\mathrm{N}$ grade), distant metastasis ( $\mathrm{M}$ grade), TNM Stages, vascular invasion and FAT4 expression in GC tissue samples (Table 3). FAT4 methylation gradually increased from $14.0 \%$ to $53.8 \%$ in patients with lymphatic metastasis (N0 to N4) and from 7.1\% (TNM Grade I) to $34.6 \%$ (TNM Grade IV, $P<0.05$ ). The GC patients with lymph node metastasis exhibited higher FAT4 methylation than patients without lymph node metastasis $(38.2 \%$ vs. $14.0 \%, P<0.05)$. Moreover, patients with distant metastasis demonstrated higher FAT4 methylation than patients without distant metastasis $(57.6 \%$ vs. $17.5 \%$, $P<0.05)$.

\section{Association between $F A T 4$ promoter methylation status and GC prognosis}

We analyzed the association between FAT4 methylation status and prognosis of GC patients. In the cohort of 173 patients, the overall survival time was $33.69 \pm 1.32$ months and the overall survival rate was $12.7 \%$. The median survival time in patients showing FAT4 methylation was lower than in patients with unmethylated FAT4 $(27.00 \pm 4.20$ months vs. $40.00 \pm 1.72$ months, $P<0.05$ ). The 5-year survival rate of the methylated FAT4 patients was lower than that of the unmethylated FAT4 patients $\left(9.3 \%\right.$ vs. $14.3 \%, X^{2}=9.238, P=0.0024$, Figure 


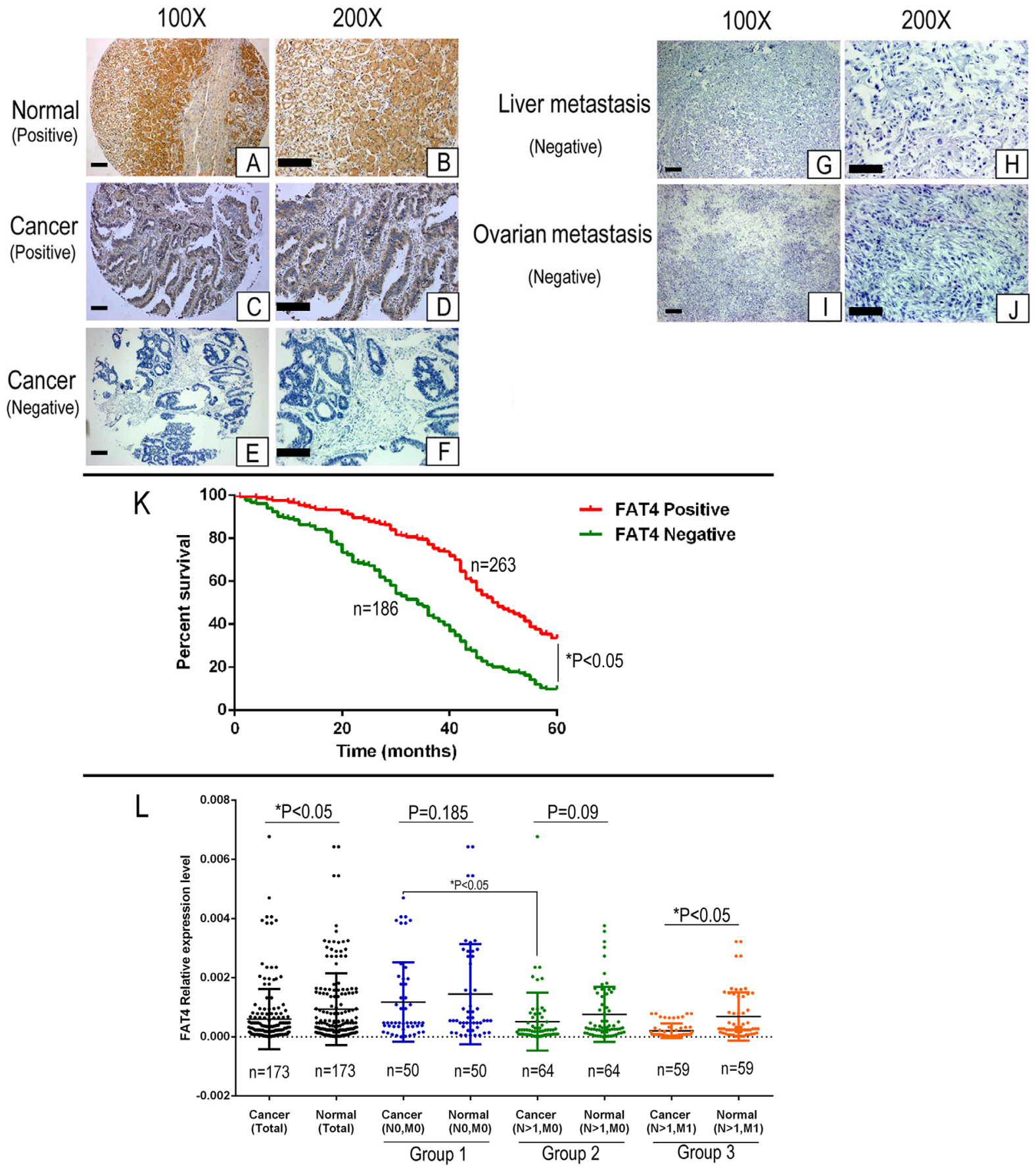

Figure 1: Immunohistochemical analysis of FAT4 in GC patient tissues and Kaplan-Meier survival analysis. (AB) Representative IHC images (100X and 200X) showing positive FAT4 expression in normal gastric epithelium. Scale bar, $100 \mu \mathrm{m}$. (C-D) Representative IHC images (100X and 200X) showing positive FAT4 expression in GC patient tissue. Scale bar, $100 \mu \mathrm{m}$. (EF) Representative IHC images (100X and 200X) showing negative FAT4 expression in GC patient tissue. Scale bar, $100 \mu \mathrm{m}$. (G-H) Representative IHC images (100X and 200X) showing negative FAT4 expression in liver metastatic tissue from GC patients. Scale bar, 100 $\mu \mathrm{m}$. (I-J) Representative IHC images (100X and 200X) showing negative FAT4 expression in ovarian metastatic tissue from GC patients. Scale bar, $100 \mu \mathrm{m}$. (K) Kaplan-Meier analysis showing survival curves of GC patients (n=449) with high (red) or low/no (green) FAT4 expression Note:" denotes P $<0.05$ compared to high FAT4 expressing GC patients. (L) qRT-PCR analysis of FAT4 mRNA expression in 173 paired GC and adjacent noncancerous patient tissue samples. FAT4 was down-regulated in the tumors than adjacent noncancerous tissues $(\mathrm{n}=173, P<0.05)$. We classified the patient samples $(\mathrm{n}=179)$ based on lymph node and distant metastases status into three groups namely, group $1(\mathrm{~N} 0, \mathrm{M} 0, \mathrm{n}=50)$, group $2(\mathrm{~N}>1, \mathrm{M} 0, \mathrm{n}=64)$ and group $3(\mathrm{~N}>1, \mathrm{M} 1, \mathrm{n}=59)$. There is no difference of FAT4 expression between cancer tissue and adjacent noncancerous tissues in group1 and group2 $(P>0.05)$, but in group3 the FAT4 expression in cancer tissue is significant lower than adjacent noncancerous tissues $(P<0.05)$. FAT4 expression was significant lower in cancer tissues from group 2 and group3 patients than group 1 patients $(P<0.05)$. 
Table 2: Multivariates analysis as determined by Cox regression analysis in 449 GC patients

\begin{tabular}{lcccc}
\hline \multirow{2}{*}{$\begin{array}{l}\text { Clinicopathological } \\
\text { parameters }\end{array}$} & \multicolumn{9}{c}{$\mathbf{9 5 \% \text { Confidential interval }}$} & Hazard ratio & \multirow{2}{*}{$\boldsymbol{P}$ value } \\
\cline { 2 - 3 } & Lower & Upper & & \\
\hline Distant metastasis & 1.695 & 1.038 & 4.447 & 0.035 \\
TNM stage & 1.217 & 2.133 & 11.126 & 0.001 \\
Lauren classification & 1.915 & 1.445 & 20.393 & 0.000 \\
Lymph node metastasis & 1.591 & 1.079 & 5.504 & 0.019 \\
FAT4 expression & 1.043 & 1.685 & 5.310 & 0.021 \\
\hline
\end{tabular}
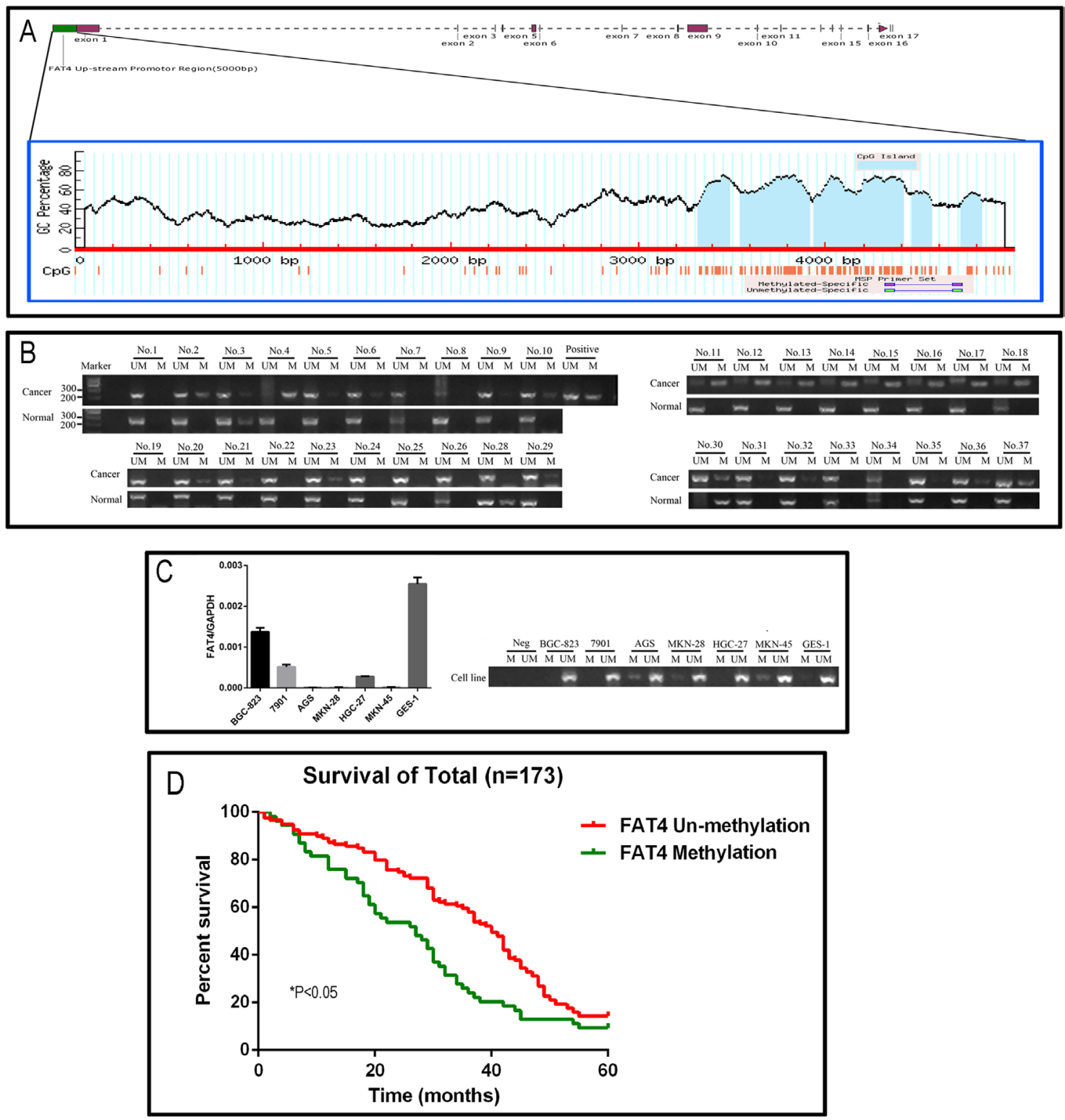

Figure 2: Analysis of FAT4 promoter methylation status and its association with GC. (A) Schematic representation of the $4.5 \mathrm{~kb} \mathrm{CpG}$ island region in the human FAT4 gene promoter. (B) The representative picture of the Methylation specific PCR analysis of FAT4 promoter region in GC tissues. The samples Nos. 2, 4 and 11-18 show high FAT4 methylation and Nos. 6, 9, 10 and 32 show low methylation. (C) FAT4 expression levels and methylation specific PCR analysis of FAT4 promoter region in GC cell lines. (D) KaplanMeier analysis comparing survival curves of $173 \mathrm{GC}$ patients with and without FAT4 methylation. Patients without FAT4 methylation (red) show increased survival than patients with FAT4 methylation $\left({ }^{*} P<0.05\right)$. 
Table 3: Association between FAT4 methylation status and clinicopathological factors

\begin{tabular}{|c|c|c|c|c|}
\hline \multirow{2}{*}{ Clinical parameters } & \multicolumn{2}{|c|}{ FAT4 } & \multirow{2}{*}{$\mathrm{t} / \chi^{2}$} & \multirow{2}{*}{$\boldsymbol{P}$} \\
\hline & Unmethylation & Methylation & & \\
\hline Age(yrs) & $61.00 \pm 13.14$ & $62.50 \pm 14.90$ & & \\
\hline Gender & & & 0.116 & 0.733 \\
\hline Male & $91(69.5 \%)$ & $40(30.5 \%)$ & & \\
\hline Female & $28(66.7 \%)$ & $14(33.3 \%)$ & & \\
\hline Location & & & 4.390 & 0.111 \\
\hline Proximal & $12(52.2 \%)$ & $11(47.8 \%)$ & & \\
\hline Middle & $45(67.2 \%)$ & $22(32.8 \%)$ & & \\
\hline Distal & $62(74.7 \%)$ & $21(25.3 \%)$ & & \\
\hline Size & & & 1.117 & 0.291 \\
\hline$<5 \mathrm{~cm}$ & $61(72.6 \%)$ & $23(27.4 \%)$ & & \\
\hline$\geq 5 \mathrm{~cm}$ & $58(65.2 \%)$ & $31(34.8 \%)$ & & \\
\hline Histology type & & & 6.962 & 0.073 \\
\hline Papillary adenocarcinoma & $1(20.0 \%)$ & $4(80.0 \%)$ & & \\
\hline Tubular adenocarcinoma & $96(72.2 \%)$ & $37(27.8 \%)$ & & \\
\hline Mucinous adenocarcinoma & $4(57.1 \%)$ & $3(42.9 \%)$ & & \\
\hline Signet-ring cell carcinoma & $18(64.3 \%)$ & $10(35.7 \%)$ & & \\
\hline Lauren classification & & & 13.917 & 0.000 \\
\hline Diffuse type & $55(85.9 \%)$ & $9(14.1 \%)$ & & \\
\hline Intestinal type & $64(58.7 \%)$ & $45(41.3 \%)$ & & \\
\hline Differentiation & & & 2.413 & 0.299 \\
\hline Well & $0(0.00 \%)$ & $1(100.0 \%)$ & & \\
\hline Moderately & $32(66.7 \%)$ & $16(33.3 \%)$ & & \\
\hline Poorly & $87(70.2 \%)$ & $37(29.8 \%)$ & & \\
\hline Invasion Depth (T Grade) & & & 11.119 & 0.011 \\
\hline $\mathrm{T} 1$ & $15(93.8 \%)$ & $1(6.3 \%)$ & & \\
\hline $\mathrm{T} 2$ & $29(74.4 \%)$ & $10(25.6 \%)$ & & \\
\hline $\mathrm{T} 3$ & $64(68.1 \%)$ & $30(31.9 \%)$ & & \\
\hline $\mathrm{T} 4$ & $11(45.8 \%)$ & $13(54.2 \%)$ & & \\
\hline Lymphatic Metastasis (N Grade) & & & 20.846 & 0.000 \\
\hline N0 & $43(86.0 \%)$ & $7(14.0 \%)$ & & \\
\hline $\mathrm{N} 1$ & $26(78.8 \%)$ & $7(21.2 \%)$ & & \\
\hline $\mathrm{N} 2$ & $26(68.4 \%)$ & $12(31.6 \%)$ & & \\
\hline N3 & $24(46.2 \%)$ & $28(53.8 \%)$ & & \\
\hline Distant metastasis (M Grade) & & & 29.093 & 0.000 \\
\hline M0 & $94(82.5 \%)$ & $20(17.5 \%)$ & & \\
\hline M1 & $25(42.4 \%)$ & $34(57.6 \%)$ & & \\
\hline
\end{tabular}

(Continued) 


\begin{tabular}{|c|c|c|c|c|}
\hline \multirow{2}{*}{ Clinical parameters } & \multicolumn{2}{|c|}{ FAT4 } & \multirow{2}{*}{$t / \chi^{2}$} & \multirow{2}{*}{$P$} \\
\hline & Unmethylation & Methylation & & \\
\hline TNM Stages & & & 32.214 & 0.000 \\
\hline $\mathrm{I}$ & $26(92.9 \%)$ & $2(7.1 \%)$ & & \\
\hline II & $51(85.0 \%)$ & $9(15.0 \%)$ & & \\
\hline III & $17(65.4 \%)$ & $9(34.6 \%)$ & & \\
\hline IV & $25(42.4 \%)$ & $34(57.6 \%)$ & & \\
\hline Lymphatic metastasis & & & 9.706 & 0.002 \\
\hline No & $43(86.0 \%)$ & $7(14.0 \%)$ & & \\
\hline Yes & $76(61.8 \%)$ & $47(38.2 \%)$ & & \\
\hline Vascular invasion & & & 11.978 & 0.001 \\
\hline No & $92(76.7 \%)$ & $28(23.3 \%)$ & & \\
\hline Yes & $26(50.0 \%)$ & $26(50.0 \%)$ & & \\
\hline FAT4 expression (IHC Result) & & & 69.677 & 0.000 \\
\hline Negative & $29(36.7 \%)$ & $50(63.3 \%)$ & & \\
\hline Positive & $90(95.7 \%)$ & $4(4.3 \%)$ & & \\
\hline
\end{tabular}

All cases were classified according to the World Health Organization's (2010) pathological classification of gastric cancer. Invasion Depth (T Grade) grade T1 includes T1a and T1b, T4 includes T4a and T4b. Lymphatic Metastasis (N Grade) grade N3 includes N3a and N3b. TNM grade I includes Ia and Ib, TNM grade II includes IIa and IIb, TNM grade III includes IIIa, IIIb and IIIc.

2D). Cox multivariate analysis indicated that TNM stage, distant metastasis, lymph node metastasis and vascular invasion were independent prognostic factors $(P<0.05)$, but, FAT4 methylation status was not an independent prognostic factor (Hazard Ratio $=0.160, P=0.689$ ).

\section{FAT4 levels determine in vitro growth and invasiveness of GC cell lines}

We knocked down FAT4 expression in the BGC823 cell line that exhibits relatively higher FAT4 expression than other GC cell lines (Figure 2C and Supplementary Figure 3B). Western blot and qRT-PCR analysis demonstrated that stable transfection with FAT4-shRNA resulted in decreasing FAT4 expression (Figure 3A). Concurrently, FAT4 expression was knocked down in BGC-823 cells by the sgRNA guided CRISPCAS9 system (Figure 3A). Analysis by xCELLigence system demonstrated higher cell growth, migration and invasiveness in BGC-823-FAT4-KO and BGC-823shFAT4 cells than BGC-823-KO-NC and BGC-823-shNC cells (Figure 3B; $P<0.05$ ). The MTT assay demonstrated that BGC-823-FAT4-KO and BGC-823-shFAT4 cells showed higher proliferation rates than BGC-823-KO-NC and BGC-823-shNC control cells (Figure 4A; $P<0.05$ ). Wound healing assay showed increased migration of
BGC-823-FAT4-KO and BGC-823-shFAT4 cells than the control cells (Figure 4B). Transwell migration and invasion assays showed increased migration and invasiveness of BGC-823-FAT4-KO and BGC-823shFAT4 cells than the controls (Figure 4C; $P<0.05$ ). These data suggested that FAT4 knockdown promoted GC cell proliferation, migration and invasion.

\section{FAT4 knockout GC cells promote tumorigenesis and lung metastasis in nude mice xenograft model}

Next, we tested the effects of FAT4 knockdown on GC tumorigenesis and invasiveness in a nude mice xenograft model by transplanting BGC-823-FAT4-KO and BGC-823-KO-NC cells.

In the subcutaneous implant models, we observed increased tumor growth in the BGC-823-FAT4-KO group than the control BGC-803-NC group (Figure 5A). Moreover, BGC-823-FAT4-KO cells showed increased tumor volume than BGC-823-KO-NC cells $(463.70 \pm$ $263.58 \mathrm{~mm}^{3}$ vs. $64.31 \pm 27.36 \mathrm{~mm}^{3}$; Figure $5 \mathrm{~B}, P<0.05$ ).

We analyzed the metastatic status in tail vein injection models after 5 weeks by PET-CT scanning. CT scans indicated the presence of lung nodules in the BGC823-FAT4-KO group (red arrow), which was absent or ill- 
Reference CACCGGAGCCCTGTACACCACCTCCACCATCGACCGCGAGAGCCTGCCCAGCGACGTGATCAA FAT4-KO-Segence CACCGGAGCCCTGTACACCA--- -- -- ---CCGCGAGAGCCTGCCCAGCGACGTGATCAA
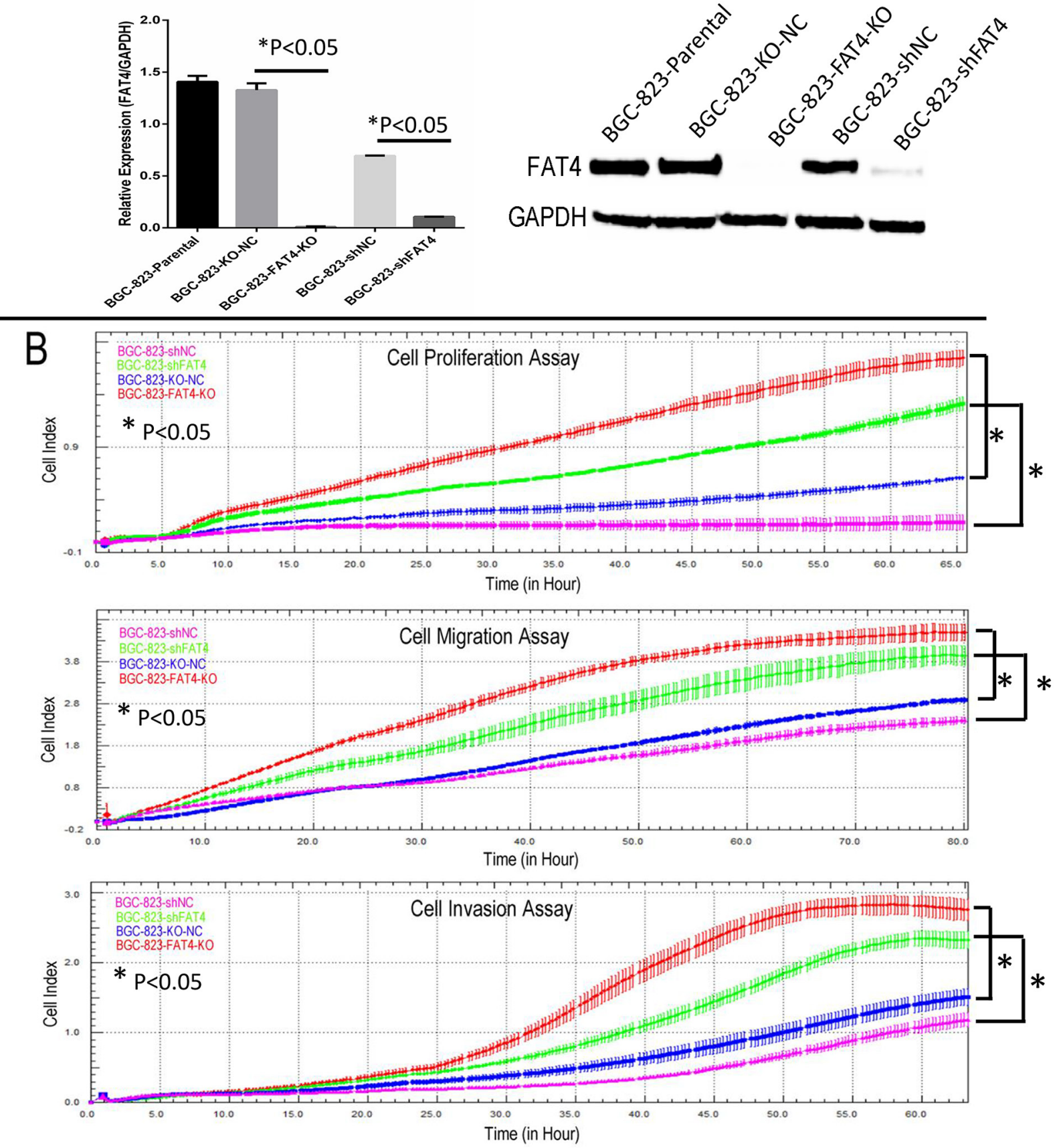

Figure 3: Real time cellular analysis of the effect of FAT4 silencing on GC cell growth, migration and invasion. (A) Schematic representation of the sgRNA binding site in FAT4 gene and the FAT4 knock-out model by CRISPR/CAS9 methodology. (B) Real Time Cellular Analysis (RTCA) showing relative cell indices of cellular growth, migration and invasion of BGC-823-FAT4-KO and BGC-823-shFAT4 cells and their corresponding controls, BGC-823-KO-NC and BGC-823-shNC cells. Note:* denotes $\mathrm{P}<0.05$ compared to corresponding controls. 

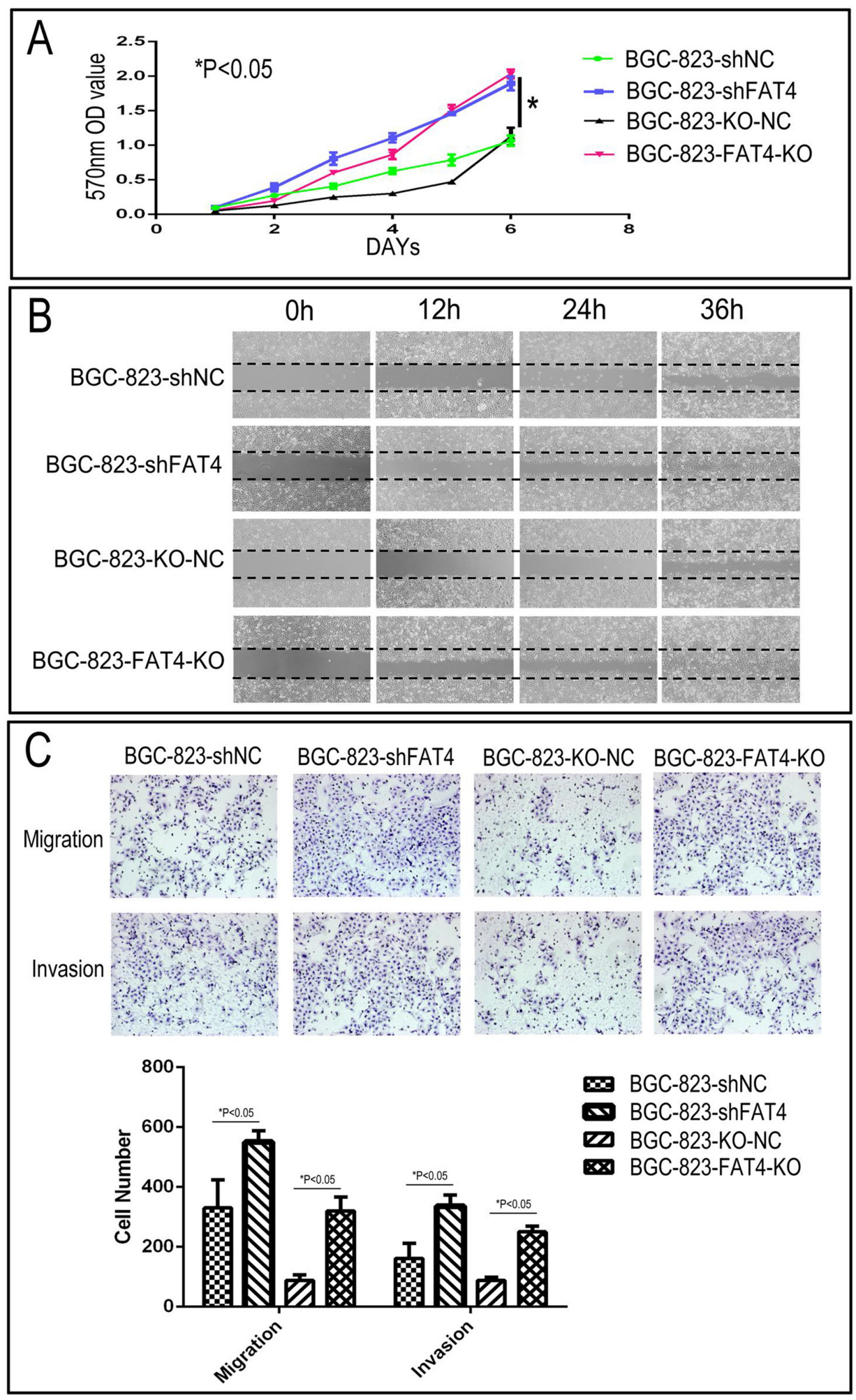

Figure 4: Effect of FAT4 silencing on cell proliferation, migration and invasion of GC cells by MTT, wound healing and Transwell migration and invasion assays. (A) MTT assay showing cell proliferation rates of BGC-823-FAT4-KO and BGC823-shFAT4 cells and their corresponding controls, BGC-823-KO-NC and BGC-823-shNC cells. As shown, FAT4 knockdown and knockout cells demonstrate higher proliferation rate than the controls ( $\left.{ }^{*} \mathrm{P}<0.05\right)$. (B) Representative images of wound healing assay showing increased migration of BGC-823-FAT4-KO and BGC-823-shFAT4 cells than their corresponding controls, BGC-823-KO-NC and BGC823-shNC cells. (C) Transwell migration and invasion assays demonstrating increased migration and invasiveness of BGC-823-FAT4-KO and BGC-823-shFAT4 cells than their corresponding controls, BGC-823-KO-NC and BGC-823-shNC cells. Data represent mean \pm SD from 3 independent experiments. ${ }^{*}$ denotes $P<0.05$ compared to corresponding controls. 
defined in the control group (Figure 6A). PET evaluation of the two groups of mice indicated high ${ }^{18} \mathrm{~F}-\mathrm{FDG}$ activity in the brain, bladder, heart and muscle tissues, but not in the lungs. Despite this observation, the BGC-823-FAT4$\mathrm{KO}$ group mice showed higher ${ }^{18} \mathrm{~F}-\mathrm{FDG}$ activity and multiple tumors in the lungs than the BGC-823-KO-NC group mice (Figure 6A; blue arrows). These findings are consistent with the presence of the macroscopic lung metastatic cancer nodules in the lung tissues (Figures 6A and 7A). Gross anatomical analysis of lung metastasis nodules showed higher and larger number of nodules in the BGC-823-FAT4-KO group than the BGC-823-KO-NC group (117.5 \pm 16.65 vs. $15.83 \pm 5.31, P<0.05$; Figure $6 \mathrm{~B})$.

Furthermore, tumors of the BGC-823-FAT4-KO group showed weak FAT4 staining and higher Ki-67 index than the BGC-823-KO-NC group (Figures 5C-5D and 7B7C). These findings were evident in both subcutaneous implant tumors and lung metastatic cancer nodules. Overall, these results indicate that loss of FAT4 expression promotes GC progression and metastasis.

\section{DISCUSSION}

Many developmental genes that are involved in cell proliferation and apoptosis regulation are deregulated in human diseases including cancers. The Fat gene family is a member of the cadherin super family, which controls cell proliferation and planar cell polarity during Drosophila development $[13,14]$. In mammals including humans, FAT family members regulate Hippo signaling pathway $[9,12,13]$. Whole-exome sequencing revealed mutations (5\%) and deletions (4\%) in FAT4 of GC cases [8]. FAT4 knockdown increased the migratory and invasive ability of breast cancer cells [9]. The Hippo pathway in mammals involves a kinase cascade that involves phosphorylation and activation of large tumor suppressor $1 / 2($ LATS1/2) by Ste20-like kinases $1 / 2$ (MST1/2). This kinase cascade inhibits the activities of two transcriptional co-activators, Yes-associated protein (YAP) and transcriptional coactivator with PDZ-binding motif (TAZ) [15]. When YAP and TAZ are activated,
A

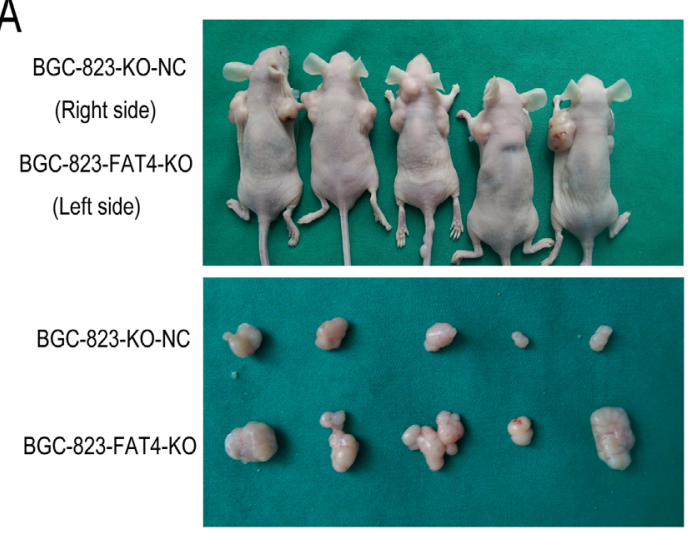

B

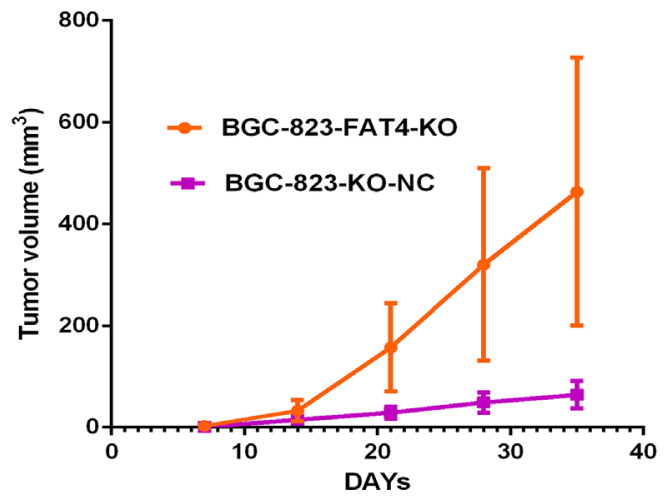

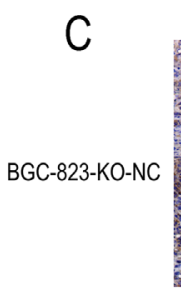

BGC-823-FAT4-KO
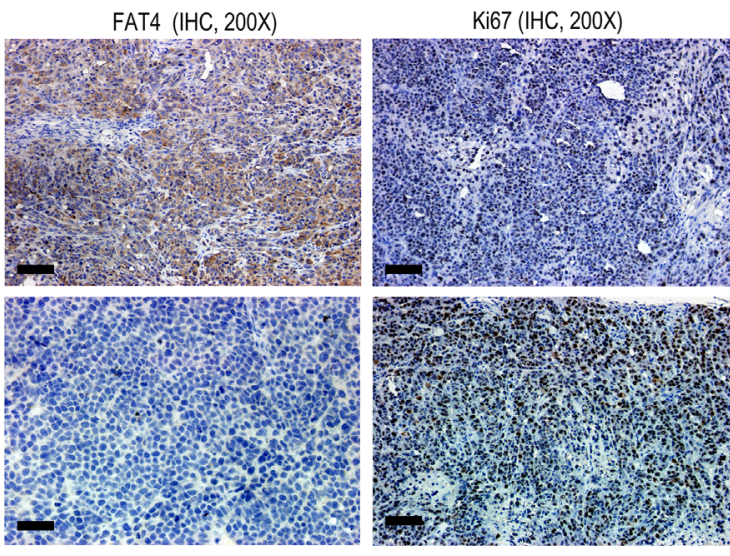

D

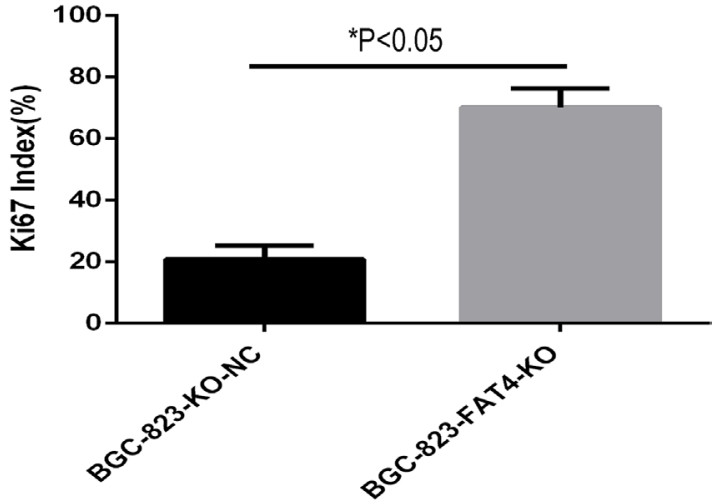

Figure 5: Effect of FAT4 knockout on GC tumorigenesis in the nude mice xenograft model. (A) Nude mice xenografted $(\mathrm{n}=5)$ with BGC-823-FAT4-KO cells (left side) show increased tumor growth (size) than tumors in mice xenografted with control BGC803-NC cells (right side). (B) BGC-823-FAT4-KO cell derived xenograft tumors show increased tumor volume than control BGC-823-KONC cells $\left({ }^{*} P<0.05\right)$. The data points represent mean tumor volume \pm SD measured on the indicated days. (C) Representative images show IHC staining of BGC-823-KO-NC and BGC-823-FAT4-KO derived xenograft tumors with antibodies against FAT4 and Ki-67. Scale bar, $100 \mu \mathrm{m}$. (D) Histogram plots show percent Ki-67 positive cells in BGC-823-KO-NC and BGC-823-FAT4-KO derived xenograft tumors. As shown, the Ki-67 index is higher in the BGC-823-FAT4-KO derived tumors than BGC-823-KO-NC derived tumors. Data represent mean \pm SD from 3 independent experiments. ${ }^{*}$ denotes $\mathrm{P}<0.05$ compared to control. 

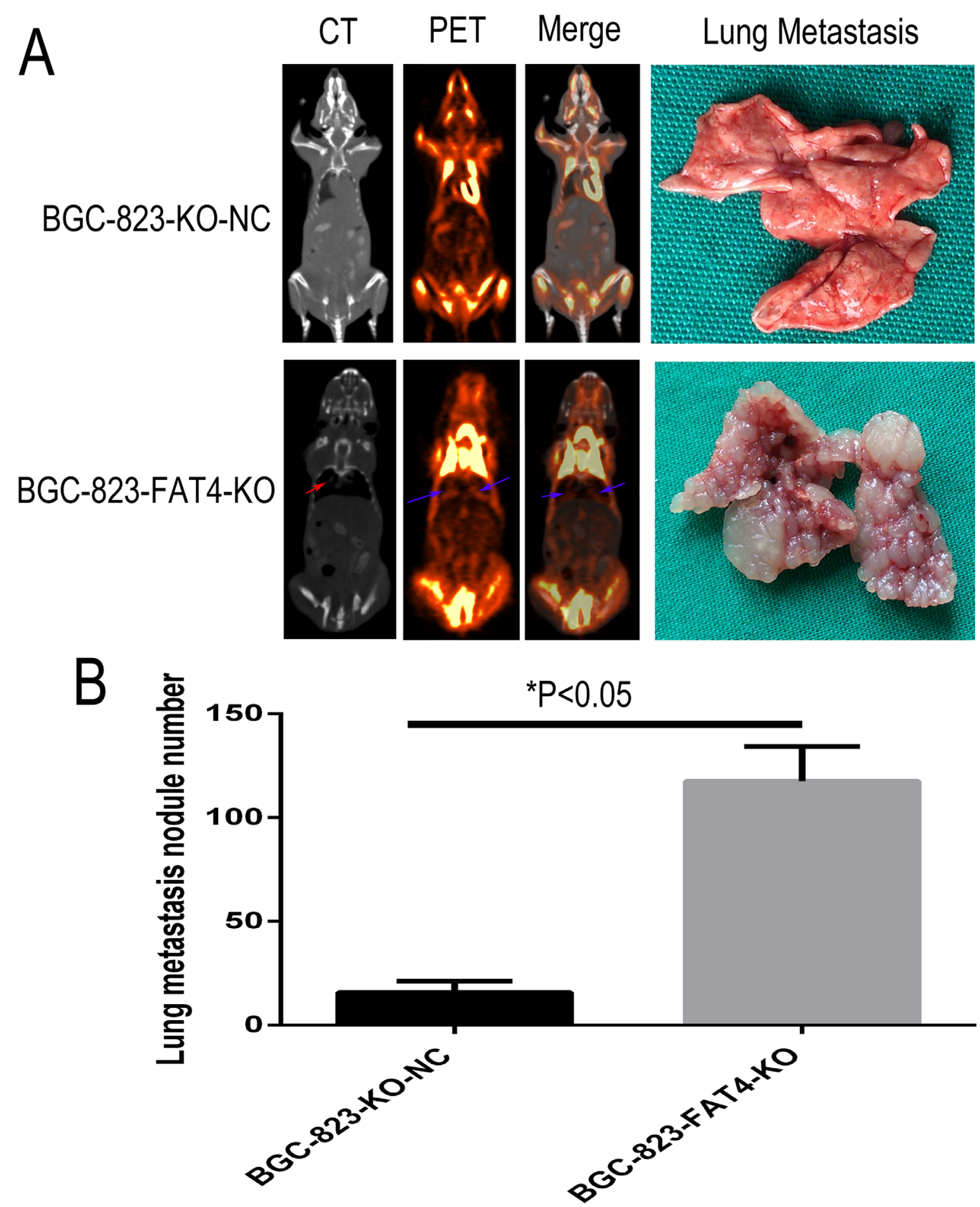

Figure 6: Effect of FAT4 knockout on lung metastasis of GC cells in the nude mice model using PET-CT scans. (A) PCTCT scan analysis showing metastasis status at 5 weeks after tail vein injection of BGC-823-FAT4-KO and BGC-823-KO-NC cells into nude mice. CT scan images (left of A) show tumor nodules in the lungs from BGC-823-FAT4-KO group (indicated by red arrow), whereas tumor nodules are absent or less clearly defined in the lungs of the control group. PET scan image (left central of A) and merge analysis image (right central of A) show higher ${ }^{18} \mathrm{~F}-\mathrm{FDG}$ activity in the lungs of BGC-823-FAT4-KO group than the BGC-823-KO-NC group (indicated by blue arrows). The lung metastasis histology figures (top right of A) shown fewer number of lung metastasis nodes in BGC-823-KO-NC, but the numbers of lung metastasis nodes are dramatically increased in BGC-823-FAT4-KO (below right of A). (B) Histogram plots show increased number of metastatic tumor nodules in the BGC-823-FAT4-KO group mice (n=5) than BGC-823-KO-NC group ( $\mathrm{n}=5$ ). Data represent mean $\pm \mathrm{SD}$ from 3 independent experiments. " denotes $P<0.05$. 
they translocate into the nucleus and induce expression of a wide range of genes that regulate tissue homeostasis and organ size control [16]. Dysregulation of the Hippo pathway is associated with cancer development [17]. Mao $\mathrm{Y}$ et al showed that the planar cell polarity complex was composed of transmembrane cadherins, Fat and Dachsous, which regulated the Hippo pathway [18]. In Drosophila, Dachsous binds to Fat, which activates the Hippo pathway by inhibiting the interaction between Zyxin and warts (Wts) (17). In vertebrates, the homolog of Fat is FAT4.

In the present study, loss of FAT4 expression was associated with poor prognosis, tumor size, invasion depth, vascular invasion, lymph node and distant metastases. FAT4 mRNA levels were lower in GC tumor samples than adjacent noncancerous tissues. We observed gradual decrease in FAT4 expression as tumor progressed from a non-metastatic state to tumor associated with lymph node and distant metastasis. FAT4 expression was similar between cancer and adjacent noncancerous tissues in patients without lymph node metastasis. But, FAT4 expression was lower in cancer tissues than adjacent normal tissues in patients with distant metastasis. Our data is in accordance with previous studies that demonstrate the tumor suppressive role of FAT4 in various cancers [9, 19].

In addition to point mutations, epigenetic modifications such as gene methylation inactivate tumor suppressor genes in tumors are very common [20-22]. Methylation of FAT4 has been reported previously in breast cancer [9]. In the present study, FAT4 promoter was methylated in $31.2 \%$ of GC patients. Methylation of the FAT4 promoter correlated with FAT4 expression levels. Patients with lymph node and/or distant metastases showed higher methylation at the FAT4 promoter than patients without metastasis. Therefore, high methylation at the FAT4 gene promoter was associated with poor prognosis for GC patients. The FAT4 promoter region
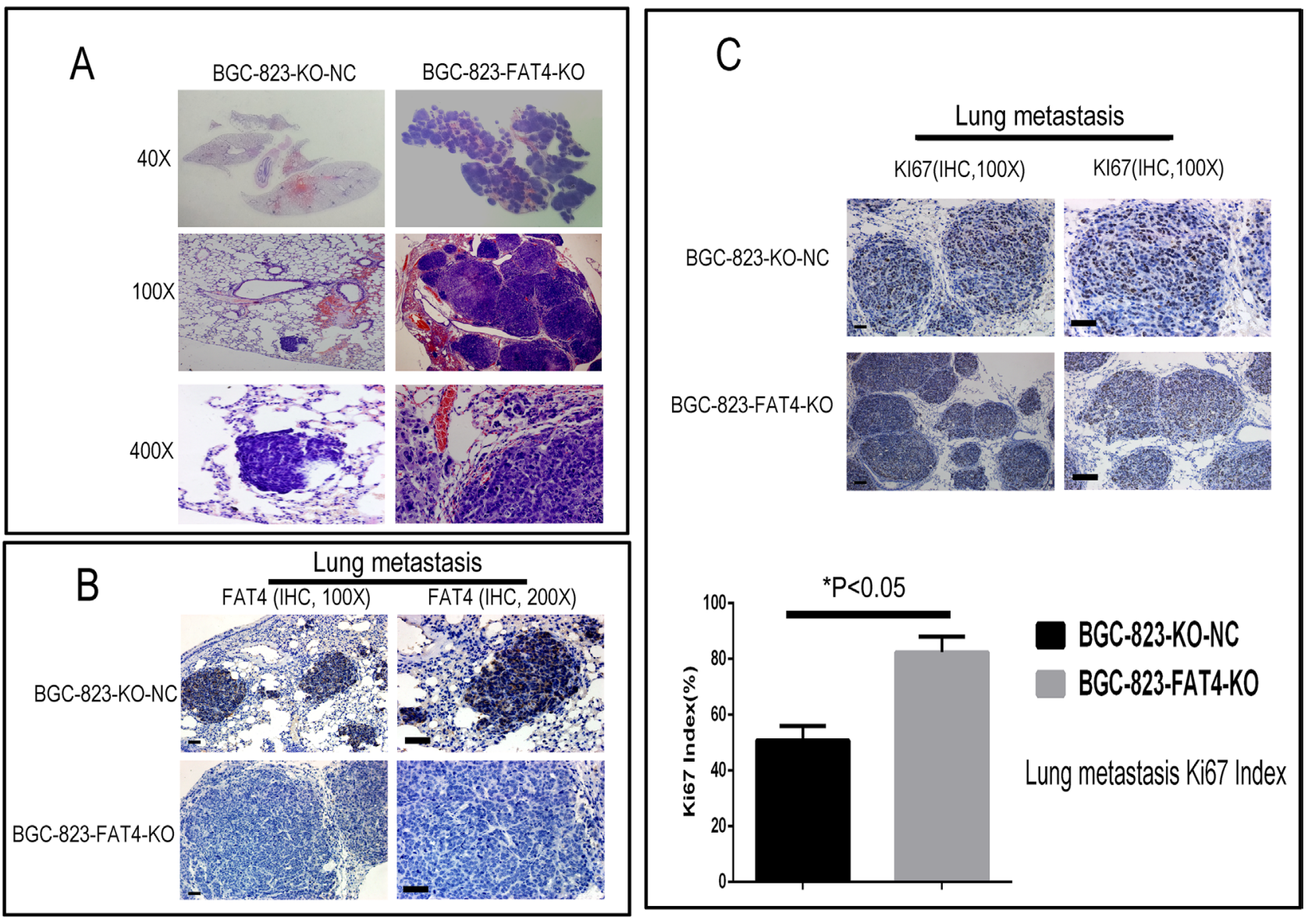

Figure 7: H\&E and IHC staining of metastatic lung nodules in the nude mice model. (A) Representative images (40X, 100X and 400X) of H\&E stained lung sections from nude mice injected via the tail vein with BGC-823-FAT4-KO and BGC-823-KO-NC cells. (B) Representative images show IHC staining with anti-FAT4 antibody of lung sections from BGC-823-FAT4-KO and BGC-823-KO-NC group mice. FAT4 is positive staining in the lung metastatic tumor nodules of BGC-823-KO-NC group, whereas it negative expresses in BGC-823-FAT4-KO group. Scale bar, $100 \mu \mathrm{m}$. (C) Histogram shows Ki-67 expression and index (\%) in lungs of the BGC-823-FAT4-KO and BGC-823-KO-NC group mice. As shown, the BGC-823-FAT4-KO group shows higher Ki-67 index (\%) than BGC-823-KO-NC group. Data represent mean \pm SD from 3 independent experiments. Scale bar, $100 \mu \mathrm{m} .{ }^{*}$ denotes $P<0.05$ compared to control. 
was also methylated to various degrees in different GC cell lines. S-azacytidine, a demethylating agent increased FAT4 expression in GC cell lines. This indicated that the decrease in FAT4 expression was partly due to promoter methylation as previously reported in breast cancer [9] and few stage I lung adenocarcinoma patients [23].

These data suggested that decrease in FAT4 levels was critical for GC progression. The reversal of FAT4 expression alone could not completely block the tumorigenic properties of the GC cells because of acquired genetic changes during their transformation. We demonstrated the critical role of FAT4 in GC progression by showing that FAT4 knockout BGC-823 cells showed increased tumor growth and progression, both in vitro and in vivo.

In conclusion, our study demonstrates that decreased FAT4 expression is associated with GC progression resulting in poor prognosis. Moreover, FAT4 promoter methylation decreases FAT4 expression. Thus, our study demonstrates that FAT4 is a potential prognostic marker and therapeutic target for GC patients.

\section{MATERIALS AND METHODS}

\section{GC patient samples}

We performed tissue microarray and immunohistochemical analyses on FFPE samples obtained from 449 GC patients enrolled from January 1998 to January 2004 at the Zhejiang Provincial People's Hospital. The patients were followed up for over 5 years until December 2009. The survival time was estimated from the date of surgery to the end of the follow-up period or the date of death due to carcinoma recurrence. The patients did not receive radiotherapy and/or chemotherapy prior to surgery. We obtained written informed consent from all patients prior to analysis. The age of the GC patients ranged from 17 to 80 (median age: $59.3 \mathrm{y}$ ). The patients were classified according to the World Health Organization pathological classification (2010) of tumors. The clinicopathological characteristics of the GC patients are summarized in Table 1. The core tumor area (tumor occupying $>50 \%$ ) was determined by the pathologist based on H\&E staining of the 449 FFPE GC tissue wax blocks. Subsequently, 35 (approximately $2 \mathrm{~mm}$ diameter) individual paraffin embedded GC blocks were arranged in a recipient paraffin microarray block (tissue array blocks) using a trephine. Finally, 13 tissue array blocks were generated from the 449 GC cases. Each block contained more than three internal controls consisting of normal gastric mucosa.

FAT4 loss of function was investigated in 173 frozen GC tissue samples. Methylation analysis was conducted on RNA and DNA samples isolated from the 449 samples. This cohort included 50 cases without lymph node and distant metastases, 59 cases with distant metastases (40 with liver metastasis and 19 with ovary metastasis) and 64 cases with lymph node metastasis alone. The clinicopathological characteristics of the GC patients are summarized in Table 3.

\section{Immunohistochemical staining and evaluation}

The GC patient tumor tissue microarray (TMA) and the whole sections of distant metastases tissues were used to analyze FAT4 by IHC. The sections were deparaffinized, rehydrated, and subjected to antigen retrieval combined with signal detection as described previously [24]. The sections were incubated with primary FAT4 antibody (ab198905, 1:100 dilution) overnight at $4^{\circ} \mathrm{C}$. They were developed with 3, 3-diaminobenzidine (DAB) and counterstained with hematoxylin. Fat4 staining intensity was determined under a light microscope on a scale of $0-3$. The proportion of positively stained cells $(0,<5 \% ; 1,5-25 \% ; 2,26-50 \% ; 3,51-75 \% ; 4,76-100 \%)$ were independently estimated by two pathologists in the absence of clinical information as described previously $[24,25]$. The intensity and proportion scores were subsequently multiplied to obtain a composite score. A score of 0 to 3 was considered negative, whereas scores of 4 to 12 were considered positive.

\section{RNA and DNA extraction}

The E.Z.N.A. ${ }^{\circledR}$ DNA/RNA Isolation Kit (R673101, Omega Bio-tek, GA, USA) was used to simultaneously isolate genomic DNA and total RNA from 90 GC tissues and cell line extracts. Briefly, the tissue samples were initially lysed and homogenized in a denaturing buffer and the supernatant was applied to a HiBind DNA spin column that binds the DNA. The RNA pellet was dissolved and purified by a HiBind RNA spin column. The purity and concentration of the samples were measured by a NanoDrop 2000 spectrophotometer (Thermo Scientific, USA).

\section{Quantitative real-time PCR (qRT-PCR)}

FAT4 mRNA levels were analyzed by qRT-PCR. Total RNA from tissue samples and cultured cells was reverse transcribed to cDNA with PrimeScript $\mathrm{t}^{\mathrm{TM}}$ RT Master Mix (Perfect Real Time) kit (RR036A, Takara) according to manufacturer's instructions. Real-time PCR was carried out by the SYBR Green master mix kit in a CFX96 thermal cycling instrument (Bio-Rad). GAPDH was used as endogenous control. The primer sequences were as follows: FAT4-F: 5'-TATCACAAAACGCCCTTGCT-3', FAT4-R: 5'-TGGATTGTCATTGATATCCTG-3'. GAPDH-F: 5'-TGAAGGTCGGAGTCAACGG-3' and GAPDH-R: 
5'-CTGGAAGATGGTGATGGGATT-3'. The PCR cycling conditions were as follows: $95^{\circ} \mathrm{C}$ for $4 \mathrm{~min}$ followed by 40 cycles of $95^{\circ} \mathrm{C}$ for $10 \mathrm{~s}, 60^{\circ} \mathrm{C}$ for $30 \mathrm{~s}$ and $72^{\circ} \mathrm{C}$ for $30 \mathrm{~s}$. The relative expression was determined by the $2^{-\Delta \Delta \mathrm{Ct}}$ method with the $\mathrm{Ct}$ values obtained from the melting curves.

\section{Methylation specific PCR and analysis}

The methylation status of FAT4 gene was determined by methylation specific PCR (MSP) using bisulfite modified genomic DNA as the template. Bisulfite modified genomic DNA was prepared with the EZ DNA Methylation-Gold ${ }^{\text {TM }}$ Kit (Zymo Research, D5006, CA, USA) according to the manufacturer's protocol. MSP was carried out in a $50 \mu \mathrm{l}$ reaction mixture containing $5 \mu \mathrm{l}$ of bisulfite treated DNA. The MSP assay conditions were as follows: $95^{\circ} \mathrm{C}$ for $4 \mathrm{~min}$ followed by 40 cycles of $95^{\circ} \mathrm{C}$ for $10 \mathrm{~s}, 62^{\circ} \mathrm{C}$ for $30 \mathrm{~s}$ and $72^{\circ} \mathrm{C}$ for $30 \mathrm{~s}$. The reverse primer sequence for both methylated and unmethylated templates was 5'-CCTATATCTAAAATATATAAAAAATC-3'. The forward primers for methylated and unmethylated templates were 5'-GTTTTAGCGGTTATTGTCGGC-3' and 5'-GTTTTAGTGGTTATTGTTGGT-3', respectively. After completion of the PCR, the samples were analyzed on a $1.5 \%$ agarose gel and the methylated or unmethylated PCR products ( $280 \mathrm{bp})$ were detected by the Bio-Rad ChemiDoc ${ }^{\mathrm{TM}}$ MP System.

\section{Methylation pyrosequencing}

We used pyrosequencing analysis to quantify the methylation levels in 36 pairs of GC and adjacent noncancerous tissues and 7 GC cell lines to verify the MSP results. For bisulfite conversion of the target sequences, EpitTect Bisulfite Kit (Cat No.: 59104, QIAGEN, Germany) was used according to the manufacturer's manual. Pyrosequencing was performed using a PyroMark Gold Q96 Reagents Kit (Cat No.: 972804, QIAGEN, Germany) in a PyroMark Q96 ID System (QIAGEN, Germany) according to the manufacturer's instructions. CpG site methylation was quantified by Pyro Q-CpG 1.0.9 software (QIAGEN, Germany).

In brief, double stranded PCR products were denatured with $0.1 \mathrm{M}$ sodium hydroxide $(\mathrm{NaOH})$ followed by PBS washes. It was then annealed to the sequencing primer (5'-CTACAACCCCCCTCCA-3'). The pyrosequencing reaction was started at the 3 '-end of the sequencing primer by adding $\mathrm{A}, \mathrm{T}, \mathrm{C}$, and $\mathrm{G}$ nucleotides into each sample well, one at a time. As the bases were incorporated into the growing DNA strand based on complimentary base pairing, each incorporated base resulted in light production as a result of activation of an enzymatic cascade. The light intensity was graphed in a program and analyzed by the Pyromark $\mathrm{CpG}$ software to determine average methylation at all $\mathrm{CpG}$ sites.

\section{FAT4 knock-out by the CRISPR/Cas9 system}

To knockout FAT4, sgRNA sequence, 5'GGGCAGGCTCTCGCGGTCGA-3, that targeted exon1 of FAT4 was designed with the Zhang Lab web application (http://crispr.mit.edu/). The FAT4 specific sgRNA was cloned into the pL6-U6-Bbsl_ Bbsl_gRNA scaffold plasmid (Addgene, Cambridge, USA), linearized by digestion with Bbsl (Thermo Scientific, ER1011, USA) for $30 \mathrm{~min}$ at $37^{\circ} \mathrm{C}$ and purified by gel electrophoresis. Subsequently, pair of oligonucleotide sequences was designed to target the FAT4 exon 1, namely, FAT4-KO-sgRNA-F: ACCGGGGCAGGCTCTCGCGGTCGA and FAT4-KOsgRNA-R: AAACTCGACCGCGAGAGCCTGCCC. The two oligonucleotides were annealed, phosphorylated and ligated to the linearized pL6 vector. BGC-823 GC cells were transfected in a 6-well plate with $250 \mathrm{ng}$ of pL6-U6-Bbsl_Bbsl_gRNA scaffold and/or an empty pL6-U6-Bbsl_Bbsl_gRNA scaffold plasmid and 500 ng of pXC-FLAG-hCas9 plasmid using polyethylenimine (PEI, Polyplus transfection ${ }^{\circledR}$ SA, 115-010, USA). After $48 \mathrm{~h}$, the cells were selected for 3 weeks with $1 \mu \mathrm{g}$ / $\mathrm{ml}$ puromycin. Then, single colonies were tested for authentic clones by PCR amplification of the CRISPR binding site using the following primers: forward: 5'-CCCGTTGCACACTCTATCAGTATCT-3' and reverse: 5'-TGTCTTGCACAGTCACGTTTACCT-3'. PCR products were analyzed by Sanger sequencing.

\section{FAT4 shRNA gene silencing}

The FAT4-shRNA hairpin DNA sequences (forward: 5'-CACCGCGCATTGTTAGATAGGGAAACTCGAGTT TCCCTATCTA CAATGCGCTTTTTTG-3'; reverse: 5'AGCTCAAAAAAGCGCATTGTTAGATAGGGAAACT CG AGTTTCCCTATCTAACAATGCGC-3') were annealed and cloned into pYr-1.1 vector (Yinrun Biotechnology, Changsha, China). The plasmid was linearized by BsaI restriction enzyme and transfected into BGC-823 cells with lipofectamine 3000 (Invitrogen, USA) according to manufacturer's instructions. Stable cell lines were selected with $200 \mathrm{mg} / \mathrm{ml} \mathrm{G} 418$ treatment for 3 to 4 weeks (BGC-823-shFAT4). The control cells were generated by transfecting BGC-823 cells with pYr-1.1 vector with nonsense shRNA (BGC-823-shNC).

\section{Real time cellular analysis (RTCA) of cellular growth, migration and invasion}

The xCELLigence system (ACEA Biosciences Inc., Hangzhou, China) was used in order to monitor the cellular growth. The system consisted of microtiter plates (E-plates) with integrated gold microarrays at the bottom of the wells for continuous real time and label-free measurements of cellular status by electrical impedance. 
Cell index (CI) was determined based on relative changes in impedance at different time points $(\mathrm{T})$ in comparison to baseline $\left(\mathrm{T}_{0}\right)$. In standard E-plates, the $\mathrm{CI}$ values are proportional to the number of cells attached. The kinetic profiles reflect adhesion and spreading in approximately $6 \mathrm{~h}$ following cell seeding. In the present study, BGC823-FAT4-KO, BGC-823-shFAT4 and the corresponding control cells were seeded in E-plates at a density of 5000 cells/well in 10\% FBS supplemented RPMI-1640 medium. The E-plates were transferred to the RTCA-DP instrument for automated real-time monitoring at standard incubator conditions with quadruplet readouts of CI every $30 \mathrm{~min}$ for the following 3 days.

The rates of migration and invasion were further monitored by the xCELLigence-system in cell invasion or migration (CIM) plates as previously described [26]. CIM plates have microelectronic sensors located on the underside of a microporous membrane insert. The migrating cells adhere to the sensors resulting in higher impedance and CI. For the migration assays, BGC-823-FAT4-KO, BGC-823shFAT4 and the corresponding control cells were seeded in the upper chamber of the CIM-plates in serum-free RPMI 1640 medium at a density of 40000 and 200000 cells per well for migration and invasion assays, respectively. The bottom chambers were filled with $30 \%$ FBS supplemented RPMI 1640 medium to promote migration across membranes by generating a serum gradient. For the invasion assays, the upper chambers were loaded with $30 \mu \mathrm{l}$ of 1:20 diluted matrigel to generate a 3D biomatrix film. Following cell seeding, CIM-plates were transferred to the RTCA instrument for continuous readouts during the next $70 \mathrm{~h}$. CI was registered solely by cells that migrated through the $8 \mu \mathrm{m}$ porous membranes. The data were analyzed by the RTCA software 2.0.

\section{MTT, wound healing and transwell assays}

The RTCA data of cell growth, wound healing, migration and invasion were confirmed by the MTT, wound healing and the Transwell assays as previously described [25].

\section{Western blot analysis}

Equal amounts of total tissue or cell protein samples were subjected to $10 \%$ SDS-PAGE at $150 \mathrm{~V}$ for running $1 \mathrm{~h}$ and then wet transferred overnight onto $0.45 \mu \mathrm{m}$ PVDF membranes. The membranes were blocked with 5\% nonfat dry milk in 1X PBST buffer. Then, the blots were probed with primary antibodies FAT4 (ab130076, 1:1000, Cambridge, MA, USA) and GAPDH (ab128915, 1:3000, Cambridge, MA, USA) followed by incubation with the corresponding HRP-conjugated secondary antibodies (ab205718, 1:5000, Cambridge, MA, USA). The blots were developed with enhanced chemiluminescence (ECL) reagent
(\#34077, Thermo Fisher, USA) and imaged in the Bio-Rad ChemiDoc $^{\mathrm{TM}}$ MP system(Bio-Rad Laboratories, Inc.USA).

\section{Nude mice xenografts}

Four to five week old female BALB/c athymic nude mice were purchased from Slac Laboratory Animal Co. Ltd. (Shanghai, China). They were housed in pathogenfree conditions according to our institutional guidelines. The study protocol was approved by the Animal Care committee of our institution.

The mice $(n=5)$ were simultaneously injected with BGC-823-FAT4-KO and BGC-823-KO-NC cells (2 X $10^{6}$ cells/ $100 \mu \mathrm{l}$, in the left and right flanks, respectively. Tumor size was measured every 7 days by a slide caliper and tumor volume was calculated as (length $\times$ width $\left.^{2}\right) / 2$.

To evaluate the effect of FAT4 on GC metastasis, $1 \times 10^{6}$ BGC-823-FAT4-KO and BGC-823-KO-NC cells were injected into the lateral tail vein of the mice. Metastatic lung nodules were scanned by whole-body micro-PET/CT and quantified by H\&E staining using a dissecting microscope at each endpoint. The mice were sacrificed after 30 days and subcutaneous tumor or metastatic lung tissues were rapidly collected. A portion of the tissue was stored in liquid nitrogen, whereas the remaining was fixed in $10 \%$ neutral buffered formalin and embedded in paraffin. The tissues were cut into $4 \mu \mathrm{m}$ thick sections and stained with H\&E. Some sections were subjected to IHC staining with antibodies against FAT4(NBP1-78381F, rabbit polyclonal antiFAT4 antibody, 1: 150, Novus Biologicals, Littleton, CO, USA) and Ki-67 (\#GA62661, Clone MIB-1, Ready to use, DAKO, USA). The Ki-67 proliferation index was determined by a senior pathologist by estimating the percentage of positive nuclei in 1,000 cells in hot spots.

\section{Whole-body ${ }^{18}$ F-FDG PET/CT}

Nude mice with GC xenografts were injected with approximately $0.2 \mathrm{mCi}$ of ${ }^{18} \mathrm{~F}-\mathrm{FDG}$ for $1 \mathrm{~h}$ under continuous anesthesia. The mice were placed at the centre of the Siemens Inveon combined microPETCT scanner (Siemens Preclinical Solution USA, Inc., Knoxville, TN, USA) in a prone position. MicroCT scans were conducted under the following conditions: $\mathrm{X}$-ray tube voltage: $80 \mathrm{kV}$; current intensity: $500 \mu \mathrm{A}$; exposure time: $150 \mathrm{~ms}$ and 120 rotation steps. PET static acquisition was carried out for $10 \mathrm{~min}$ and the images were reconstructed using the OSEM (ordered set expectation maximization) algorithm for 3D PET reconstruction. The images were analyzed by the Inveon Research Workplace 4.1 (Siemens, Erlangen, Germany). The standardized uptake (SUV $\mathrm{g} / \mathrm{ml}$ ) was determined as $\mathrm{SUV}=\left[\left(\mathrm{RTA} / \mathrm{cm}^{3}\right) / \mathrm{RID}\right] \times \mathrm{BW}(\mathrm{RTA}$ is the measured 
radiotracer tissue activity in $\mathrm{mCi}$; RID is the radiotracer injected dose in $\mathrm{mCi}$ and $\mathrm{BW}$ is the mouse body weight in grams).

\section{Statistical analysis}

All statistical analyses were performed with the SPSS 13.0 statistical software. Differences between groups were estimated by 2-tailed paired Student's $\mathrm{t}$ test or independent sample t-test. The relationship between FAT4 expression and clinicopathological characteristics were analyzed by the Chi-square test. Kaplan-Meier survival curves were analyzed by the logrank test. The significance of various survival related variables were assessed by Cox regression model in the multivariate analysis. $P<0.05$ was considered statistically significant.

\section{CONFLICTS OF INTEREST}

The authors declare that there are no conflicts of interest.

\section{FUNDING}

This work was supported by the National Natural Science Foundation of China (No. 81372598; No. 81071991; No. 81502090), Science and Technology Plan of Zhejiang Province (No. 2014C33G1360171), Zhejiang Provincial Natural Science Foundation of China (LY14H160039) and Medicine and Health Research Foundation of Zhejiang Province (2013KYB022).

\section{REFERENCES}

1. Chen XZ, Zhang WH, Hu JK. A difficulty in improving population survival outcome of gastric cancer in mainland China: low proportion of early diseases. Med Oncol. 2014; 31:315.

2. Dunne J, Hanby AM, Poulsom R, Jones TA, Sheer D, Chin WG, Da SM, Zhao Q, Beverley PC, Owen MJ. Molecular cloning and tissue expression of FAT, the human homologue of the Drosophila fat gene that is located on chromosome 4q34-q35 and encodes a putative adhesion molecule. Genomics. 1995; 30:207-223.

3. Mahoney PA, Weber U, Onofrechuk P, Biessmann H, Bryant PJ, Goodman CS. The fat tumor suppressor gene in Drosophila encodes a novel member of the cadherin gene superfamily. Cell. 1991; 67:853-868.

4. Saburi S, Hester I, Goodrich L, McNeill H. Functional interactions between Fat family cadherins in tissue morphogenesis and planar polarity. Development. 2012; 139:1806-1820.

5. Pan G, Feng Y, Ambegaonkar AA, Sun G, Huff M, Rauskolb C, Irvine KD. Signal transduction by the fat cytoplasmic domain. Development. 2013; 140:831-842.
6. Katoh Y, Katoh M. Comparative integromics on FAT1, FAT2, FAT3 and FAT4. Int J Mol Med. 2006; 18:523-528.

7. Shapiro L, Fannon AM, Kwong PD, Thompson A, Lehmann MS, Grübel G, Legrand JF, Als-Nielsen J, Colman DR, Hendrickson WA. Structural basis of cell-cell adhesion by cadherins. Nature. 1995; 374:327-337.

8. Zang ZJ, Cutcutache I, Poon SL, Zhang SL, McPherson JR, Tao J, Rajasegaran V, Heng HL, Deng N, Gan A, Lim $\mathrm{KH}$, Ong CK, Huang D, et al. Exome sequencing of gastric adenocarcinoma identifies recurrent somatic mutations in cell adhesion and chromatin remodeling genes. Nat Genet. 2012; 44:570-574.

9. Qi C, Zhu YT, Hu L, Zhu YJ. Identification of Fat4 as a candidate tumor suppressor gene in breast cancers. Int $\mathrm{J}$ Cancer. 2009; 124:793-798.

10. Yoshida S, Yamashita S, Niwa T, Mori A, Ito S, Ichinose M, Ushijima T. Epigenetic inactivation of FAT4 contributes to gastric field cancerization. Gastric Cancer. 2017; 20:136-145.

11. Ma L, Cui J, Xi H, Bian S, Wei B, Chen L. Fat4 suppression induces Yap translocation accounting for the promoted proliferation and migration of gastric cancer cells. Cancer Biol Ther. 2016; 17:36-47.

12. Cai J, Feng D, Hu L, Chen H, Yang G, Cai Q, Gao C, Wei D. FAT4 functions as a tumour suppressor in gastric cancer by modulating Wnt/beta-catenin signalling. Br J Cancer. 2015; 113:1720-1729.

13. Willecke M, Hamaratoglu F, Kango-Singh M, Udan R, Chen CL, Tao C, Zhang X, Halder G. The fat cadherin acts through the hippo tumor-suppressor pathway to regulate tissue size. Curr Biol. 2006; 16:2090-2100.

14. Sadeqzadeh E, de Bock CE, Thorne RF. Sleeping giants: emerging roles for the fat cadherins in health and disease. Med Res Rev. 2014; 34:190-221.

15. Janse van Rensburg HJ, Yang $X$. The roles of the Hippo pathway in cancer metastasis. Cell Signal. 2016; 28:1761-1772.

16. Lin Q, Yang W. The Hippo-YAP/TAZ pathway mediates geranylgeranylation signaling in breast cancer progression. Mol Cell Oncol. 2016; 3:e969638.

17. Mo JS, Park HW, Guan KL. The Hippo signaling pathway in stem cell biology and cancer. EMBO Rep. 2014; 15:642-656.

18. Mao Y, Mulvaney J, Zakaria S, Morgan KM, Allen S, Basson MA, Francis-West P, Irvine KD. Characterization of a Dchs1 mutant mouse reveals requirements for Dchs1-Fat4 signaling during mammalian development. Development. 2011; 138:947-957.

19. Katoh M. Function and cancer genomics of FAT family genes (review). Int J Oncol. 2012; 41:1913-1918.

20. Brenner H, Rothenbacher D, Arndt V. Epidemiology of stomach cancer. Methods Mol Biol. 2009; 472:467-477.

21. Feinberg AP, Tycko B. The history of cancer epigenetics. Nat Rev Cancer. 2004; 4:143-153. 
22. Herman JG, Baylin SB. Gene silencing in cancer in association with promoter hypermethylation. N Engl J Med. 2003; 349:2042-2054.

23. Yachida S, Jones S, Bozic I, Antal T, Leary R, Fu B, Kamiyama M, Hruban RH, Eshleman JR, Nowak MA, Velculescu VE, Kinzler KW, Vogelstein B, et al. Distant metastasis occurs late during the genetic evolution of pancreatic cancer. Nature. 2010; 467:1114-1117.

24. He XJ, Jiang XT, Ma YY, Xia YJ, Wang HJ, Guan TP, Shao QS, Tao HQ. REG4 contributes to the invasiveness of pancreatic cancer by upregulating MMP-7 and MMP-9. Cancer Sci. 2012; 103:2082-2091.
25. He XJ, Tao HQ, Hu ZM, Ma YY, Xu J, Wang HJ, Xia YJ, Li L, Fei BY, Li YQ, Chen JZ. Expression of galectin-1 in carcinoma-associated fibroblasts promotes gastric cancer cell invasion through upregulation of integrin beta1. Cancer Sci. 2014; 105:1402-1410.

26. Scrace S, O'Neill E, Hammond EM, Pires IM. Use of the $\mathrm{xCELLigence} \mathrm{system} \mathrm{for} \mathrm{real-time} \mathrm{analysis} \mathrm{of} \mathrm{changes} \mathrm{in}$ cellular motility and adhesion in physiological conditions. Methods Mol Biol. 2013; 1046:295-306. 